\title{
Community health workers as chronic care coordinators: evaluation of an Australian Indigenous primary health care program
}

\author{
Barbara Schmidt, ${ }^{1,2}$ Sandra Campbell, ${ }^{1}$ Robyn McDermott ${ }^{1,2}$
}

\author{
hronic disease contributes \\ substantially to the health gap \\ between Indigenous and non- \\ Indigenous Australians. ${ }^{1,2}$ A major challenge \\ for clinicians, health service managers and \\ funders is to provide an adequate and \\ culturally appropriate primary health care \\ (PHC) response. Interventions to manage \\ chronic disease in remote Indigenous \\ communities have been tested in the \\ research environment ${ }^{3,4}$ and documented in \\ best practice clinical guidelines, $, 5,6$ however, \\ implementation into routine practice has not \\ been consistently achieved.
}

In the mid-1990s, the MacColl Institute described the Chronic Care Model (CCM), a conceptual model that identified essential elements of a health care system and encourages high-quality chronic disease care. ${ }^{7,8}$ The Innovative Care for Chronic Conditions Framework described by the World Health Organization in 2002 represents an expansion of the CCM, and recognises a broader environment that encompasses clients, their families, health care services, and communities. ${ }^{9}$ Effective chronic disease management requires well-organised service delivery systems, supported by modern information technology that facilitates active client engagement and fosters development of self-management skills among clients. ${ }^{10}$

The health care needs of Aboriginal and Torres Strait Islander people with chronic conditions are often complex and managed in a cross-cultural context. Indigenous Health Workers (IHWs) are important members of multidisciplinary PHC teams in Aboriginal and Torres Strait Islander communities in Australia, due to their knowledge of community and

\begin{abstract} health team members and clients. with 21 stakeholders and analysed using the key themes. required.

culture and, when trained, their ability to provide regular health checks and education about health risks and self-management. ${ }^{11}$ In many developing countries, the role of community health workers has been identified as invaluable in the management and prevention of chronic health conditions. Community health workers are usually members of the local community and from the same race/ethnicity as client groups, ${ }^{12}$ which means they are well positioned to build strong community connections and educate the community about the prevention of chronic conditions. ${ }^{13}$ In the Australian Indigenous health context and the Getting Better at Chronic Care (GBACC) study reported here, the terms 'community health
\end{abstract}

Objectives: To explore how a client-centred Chronic Care model was implemented by Indigenous Health Workers (IHWs) at participating sites in a trial of IHW-led case management. To understand the experiences of engaging with the model from the perspective of the IHWs,

Methods: The review was conducted within a cluster randomised trial of the model in six remote Indigenous communities in north Queensland over 18 months. Content analysis was undertaken on 377 project records of health worker activity. Descriptive coding was used to classify issues that were grouped under key themes. Open-ended interviews were conducted

Results: Implementation of all elements of the intervention was not achieved. Key themes identified that describe the issues affecting the IHWs' capacity to implement the model were: service management, training, client engagement, clarification of IHW role and infrastructure.

Conclusions: Placing skilled and dedicated IHWs to improve care coordination is insufficient to improve chronic disease outcomes. A supportive and systematic service delivery system is also

Implications: The PHC model in remote Indigenous communities needs to be re-oriented to actively support the unique contributions of $\mathrm{IHWs}$ to chronic care coordination.

Key words: chronic care coordination, Indigenous Health Workers, diabetes, primary health care

worker' and 'Indigenous health worker' are used interchangeably.

The GBACC project in north Queensland was developed in partnership with Apunipima Cape York Health Council and Queensland Health. ${ }^{14}$ The project aimed to test the effectiveness of intensive community-based case management delivered by trained IHWs. Clients were Aboriginal or Torres Strait Islander people with poorly controlled diabetes, and at least one other chronic condition. Twelve communities in north Queensland participated in the trial; six were randomised into the intervention group and six in the wait list group. Of the intervention sites, one was managed by an Aboriginal community-controlled organisation, three

1. Centre for Chronic Disease Prevention, James Cook University, Queensland

2. Division of Health Sciences, University of South Australia

Correspondence to: Ms Barbara Schmidt, Centre for Chronic Disease Prevention, James Cook University, P.O Box 6811, Cairns, Qld 4870; e-mail: barbara.schmidt@jcu.edu.au Submitted: October 2014; Revision requested: January 2015; Accepted: July 2015

This is an open access article under the terms of the Creative Commons Attribution-NonCommercial-NoDerivatives License, which permits use and distribution in any

medium, provided the original work is properly cited, the use is non-commercial and no modifications or adaptations are made.

The authors have stated the following conflict of interest: Dr Sandra Campbell is a member of the Australian and New Zealand Journal of Public Health Editorial Committee. Aust NZ J Public Health. 2016; 40 (Suppl. 1) S107-S114; doi: 10.1111/1753-6405.12480 
were Queensland government-managed services and two sites had a partnership arrangement where the GBACC worker was employed by a community-controlled provider, but delivered the service from the government health facility. Phase one of the project (2012-13) was a pragmatic cluster randomised control trial (RCT) where the model of care was tested in the six intervention sites. The primary clinical outcome measure was a reduction in $\mathrm{HbA} 1 \mathrm{c}$ after 18 months and the study was powered to detect a clinically meaningful mean change of $0.5 \%$. A range of secondary outcome measures related to care processes, hospitalisations and quality of life. The initial results of the trial showed a modest impact on clinical outcomes, despite achieving a significant reduction in the primary outcome measure, $\mathrm{HbA} 1 \mathrm{c}$, in the intervention sites. ${ }^{15}$

Phase one included a process evaluation of the RCT. The aim was to explore how the GBACC model was implemented in each site and to understand the experiences of key stakeholders engaging with the model. During phase two, the findings from the process evaluation and results of the RCT were used to review and reflect on the project, and to plan the rollout of the model to wait-list sites during the final third phase. Here, we report on the results of the phase one process evaluation, the experiences of GBACC IHWs with implementing the GBACC model, the other PHC multidisciplinary team members and clients engaging with the model, and the key learnings from the RCT to inform future model implementation.

\section{Intervention}

The intervention was to employ experienced IHWs with a Certificate IV in Aboriginal and Torres Strait Islander Primary Health Care to deliver chronic disease management for a caseload of clients. Participants were recruited in regional and remote Indigenous communities in the Cairns, Cape York and Torres Strait regions of northern Australia. The logic of the intervention approach was that IHWs would use client engagement strategies to facilitate the development of general practitioner (GP) management plans and team care arrangements for their clients. GP management plans are action plans developed by mutual agreement between a client and their GP, with the aim of providing an organised approach to care. Health needs are identified, GP services are set out, and client actions to help manage their own condition are listed. Team care arrangements for clients with complex needs involve at least two other health care professionals. The team works together to help coordinate their clients' primary health care needs more effectively. Examples may include a pharmacist performing home medication reviews to improve medication adherence and appointments with a diabetes educator to promote lifestyle changes and client capacity for self-management. Improvements in clinical outcomes (e.g. HbA1c and blood pressure) and reductions in avoidable hospitalisations are expected to follow from this investment in whole-person care that is integrated with other health professionals ${ }^{16}$ (Figure 1).

The GBACC IHWs were employed by the local health service and managed as usual members of the PHC team. This strategy was adopted to promote integration of chronic disease activities into usual business. The IHWs had a specific caseload and a condition of funding was that their position was supernumerary to existing team members to enable the model to be tested in the community setting. The GBACC IHWs had ongoing professional support from an Indigenous Clinical Support Team (ICST) based in Cairns.

When employment began, the GBACC IHWs were provided with three weeks of orientation and intensive clinical refresher training delivered in Cairns by the ICST. The training was in a group environment to promote and support the establishment of a peer network. Training topics included research project orientation, type 2 diabetes and other chronic conditions, chronic disease guidelines, education and engagement strategies, medication management, client self-management, care planning, care coordination and work planning. Ongoing professional support for the GBACC IHWs included weekly reflective practice sessions by telephone with an ICST member, monthly video-linked meetings, and in-service training in Cairns for one week every six months. In summary, the role of the GBACC IHWs was to participate as a member of the local multidisciplinary team. They had a defined role to provide clinical and preventive care to a specified client caseload using a holistic approach with a strong home-visiting component. They were required to manage care coordination and provide advocacy to enhance access to medical, allied health and community-based services to complete GP management plans and team care arrangements. A key element of the approach to care was to deliver effective education to build self-management capacity among clients and their families.

\section{Methods}

A two-step process using two qualitative methods was used to conduct the process evaluation of the RCT. First, a review of documents generated by routine project activities was completed to understand the experience of GBACC IHWs with implementing the GBACC model. The issues reported by IHWs were coded and grouped into themes. These themes were used as the analytical framework for the interviews with community-based stakeholders to saturate the themes. The findings from both activities were synthesised to gain an understanding of the experience of the GBACC workers and community-based stakeholders with implementing the model. Figure 2 illustrates the process evaluation design.

\section{Document review}

The GBACC project routinely produces a range of documents to manage project activities and record communication between IHWs and members of the ICST. These include minutes of IHW meetings, IHW weekly reports and ICST supervisors' reports from reflective practice sessions with IHWs or site visits. The IHW weekly reports provide structure to support IHW with systematic evaluation of chronic disease care provided and to encourage self-reflection on the outcomes of their practice. The reporting template includes data about service activity related care coordination, and free text boxes to report on issues affecting their role. In the 'barriers to progress or enablers
Figure 1: GBACC Program logic.

\begin{tabular}{|c|c|c|c|c|}
\hline \begin{tabular}{l}
\multicolumn{1}{c}{1} \\
GP Management \\
Plans \& Team \\
Care \\
Arrangements
\end{tabular} & \begin{tabular}{l}
\multicolumn{1}{c}{2} \\
Annual \\
Cycle \\
of Care
\end{tabular} & $\begin{array}{c}3 \\
\text { Therapeutic } \\
\text { actions }\end{array}$ & $\begin{array}{l}\quad 4 \\
\text { Improved clinical } \\
\text { outcomes (e.g. } \\
\text { HbA1c, blood } \\
\text { pressure } \\
\text { lipids) }\end{array}$ & $\begin{array}{c}5 \\
\text { Reduced } \\
\text { diabetes-related } \\
\text { hospitalisations }\end{array}$ \\
\hline
\end{tabular}


to success' section of the report, the IHWs document internal or individual client issues affecting their practice over the past week. The 'community' section is used to record any events that affect service delivery, e.g. funerals or sporting carnivals. The report is used to inform weekly reflective practice discussion with the ICST to build the IHW' capacity to solve problems and to identify opportunities for better engagement with the client and other service providers. The ICST supervisor's report records the key points from the reflective practice discussions and outcomes to be followed up at the next session. The IHW meeting minutes record the outcome of monthly discussions among IHWs about issues affecting them. Project records provide a robust data source to explore dayto-day service delivery and factors that affect the IHWs, from their perspective, with data collected in real time.

All the GBACC IHW weekly reports, ICST supervision reports and IHW meeting minutes for a 12-month period from the commencement of the intervention in March 2012 were included in the document review. This period covers the establishment and implementation phases of the GBACC model in each community.

\section{Semi-structured interviews}

The experiences of local community stakeholders relating to implementation and delivery of the GBACC intervention were explored through semi-structured interviews at each intervention site. The interviews were conducted in each community between 24 June and 30 August 2013 by an Aboriginal researcher who was not involved with the GBACC project, but was familiar with Indigenous health in far north Queensland.

The study used purposive sampling, guided by explicit selection criteria to ensure that respondents from three important categories in each of the participating communities were included: i) GBACC clients; ii) clinical and allied health staff; and iii) supervisory health service staff. The sampling approach was intended to provide variation to bring together a diverse range of thoughts and experiences. For example, the sampling among clients aimed to include'negative' cases (people who may have benefited from the care offered by the trial but, for various reasons, did not fully participate or benefit). Table 1 explains the stakeholders in each category.

Health service stakeholders were identified with the assistance of the GBACC Trial

Figure 2: Process evaluation design.

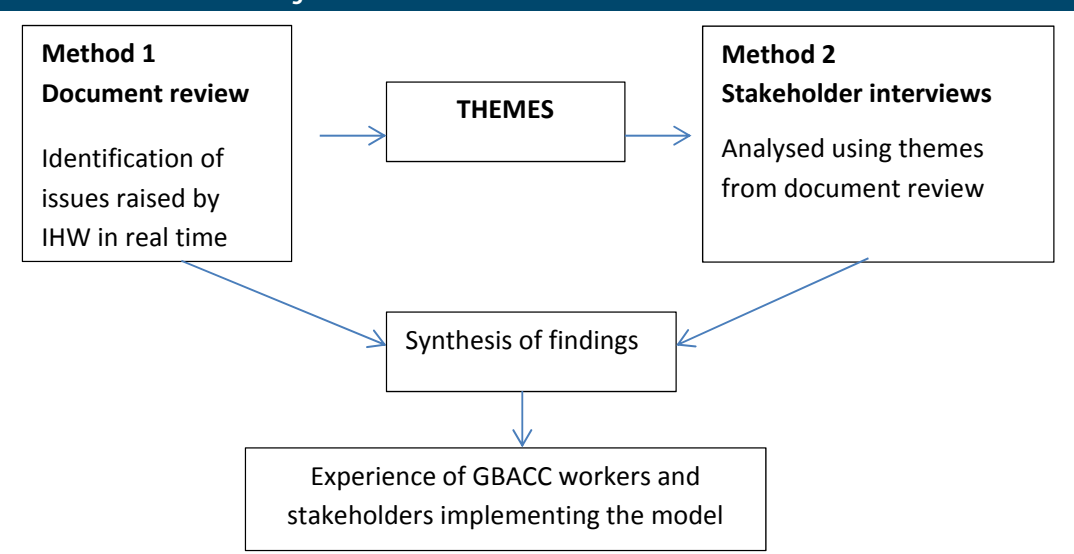

Manager and were approached directly by the interviewer. Clients were contacted with the assistance of the GBACC IHW or another IHW from the PHC team and invited to participate in an interview. Prior to any data being collected, plain language explanations about this component of the trial were provided to interviewees and informed written consent obtained.

Data was collected by face-to-face, openended, narrative interviews according to interview guides developed in consultation with the GBACC research team. The interview guides varied between participant categories; however, all participants were asked to describe and critique chronic disease care in their community. Client interviews included questions about home visiting, their access to information about their health condition and their satisfaction with care. Community PHC providers and health service supervisory staff were asked about how they viewed the role of GBACC IHWs and how well that role had been integrated with usual health service practice. Interviews were audio-recorded with permission of the participant and transcribed independently.

\section{Data analysis}

Data from the document review was analysed manually in Word tables. Free text from the 'barriers to progress or enablers for success' sections of IHW weekly reports and the notes from the 'topics' and 'key points for discussion' sections of the ICST supervision reports for the same week were extracted and inserted into the adjacent cells of the Word table. There was no capacity to analyse data from the 'community' section of the report. Arranging the data in tables enabled information from the IHW and ICST to be viewed simultaneously in chronological order. Separate tables were established for each community. The tables and the minutes of the IHW monthly meetings were reviewed by one researcher (BS) to get an understanding of the types of information contained in the documents. The review revealed that the IHW weekly reports were usually the primary source of information about issues of concern to IHWs, which were then shared at the IHW monthly meetings if the IHW felt comfortable talking about the issue. Therefore, IHW meeting minutes were excluded from further analysis.

An emic approach used in grounded theory studies was applied to analyse and code the documents to enable the IHWs' voices to define the issues of interest. Content analysis was completed by the researcher (BS) using open coding based on key words that categorised the issues raised by the IHWs and ICST in reports. The issues were then grouped into themes to provide the lens for analysis of the stakeholder interviews. The analysis of the interviews was conducted by the second researcher (SC). Passages of the transcripts

\begin{tabular}{|c|c|c|}
\hline $\begin{array}{l}\text { Category } \\
\text { No. }\end{array}$ & Category & Characteristics of stakeholders interviewed \\
\hline 1 & Clients & A sub-sample of participant clients from intervention sites. \\
\hline 2 & $\begin{array}{l}\text { Clinical \& allied health } \\
\text { staff }\end{array}$ & $\begin{array}{l}\text { Health care providers whose professional role involves the provision of chronic disease } \\
\text { management to participants falling within Category 1. The professions of participants included } \\
\text { IHWs (excluding GBACC IHWs), nurses, medical officers and allied health staff (e.g. diabetes } \\
\text { educators). }\end{array}$ \\
\hline 3 & $\begin{array}{l}\text { Supervisory health } \\
\text { service staff }\end{array}$ & $\begin{array}{l}\text { Health service management staff associated with primary health care centres located in the } \\
\text { participating communities. This category included nurses in supervisory positions (e.g. charge } \\
\text { nurses or directors of nursing) not providing clinical care to participants in Category 1. }\end{array}$ \\
\hline
\end{tabular}


that contributed data to the themes were coded manually and then collated under theme headings. Once the analysis of the interviews was completed, the data was discussed and synthesised by the researchers together and there was some rationalisation of theme categories to present the results.

The GBACC process evaluation received ethics approval from the University of South Australia, the University of Queensland, and the Far North Queensland Human Research Ethics Committees with support from the peak community-controlled services organisation, Apunipima Cape York Health Council.

\section{Results}

A total of 388 project documents were reviewed. Eleven project documents (IHW monthly meeting minutes) were excluded because data saturation was reached. In total, 377 documents were included in the document review analysis. A total of 21 face-to-face interviews from participating communities were included (Table 2). All the supervisory staff approached to be interviewed agreed to participate. Four clinical and allied health staff were approached and did not participate (three medical staff were too busy and an allied health professional had relocated to a non-participating community and declined participation when contacted by phone). The GBACC IHWs were invited but only two of six agreed. A decision was taken to exclude the IHW interviews from the analysis because they revealed no new information in addition to the comprehensive real time data about IHW views and concerns available from project records included in the document review. Five clients of 15 who were approached did not participate. There was an intention to include enrolled clients who were obviously not engaged with the project in the interview process; however, of the three approached, none agreed and two clients did not present at a pre-arranged time and place.
Content analysis identified a range of themes evident in both datasets. Enablers and barriers to model implementation are presented under the theme headings: 1) service management; 2 ) training; 3 ) client engagement; 4) clarification of GBACC IHW care coordination role; 5) infrastructure; and 6) team approach. The results are ordered so that the experiences of GBACC IHWs (identified through the document review) are generally presented first (in roman text, indented), followed by stakeholder perspectives generated by the face-to-face interviews (italics, indented).

\section{Service management}

Service management includes issues that arose from staff management practices during implementation of the model. Key sub-themes were: quarantining the GBACC IHW role to case management of GBACC clients; onsite supervision of trainee IHWs; and responsibilities around day-to-day supervision of GBACC IHWs.

In their weekly reports, GBACC IHWs at three sites consistently reported staff shortages in their respective clinics as a barrier to effective model implementation. They were regularly required to assist with duties outside their case management responsibilities. For example:

Unable to perform duty of care to the client due to staff shortages. (GBACC IHW: Community 6)

Due to limited staff - unable to visit clients. (GBACC IHW: Community 3)

This situation arose in smaller communities where the GBACC IHW is employed parttime and the overall health team has fewer members. Interview participants articulated the tensions experienced by GBACC IHWs in their daily practice as they attempted to fulfil community expectations and obligations to provide care, regardless of their care coordination role:

\begin{tabular}{|c|c|c|c|c|c|c|c|}
\hline \multirow[b]{2}{*}{ Community } & \multicolumn{2}{|c|}{ Documents } & \multirow[t]{2}{*}{ Total } & \multicolumn{3}{|c|}{ Interviews ${ }^{\mathrm{a}}$} & \multirow[b]{2}{*}{ Total } \\
\hline & $\begin{array}{c}\text { Weekly IHW } \\
\text { report }\end{array}$ & $\begin{array}{c}\text { Supervisor } \\
\text { report }\end{array}$ & & Clients & $\begin{array}{l}\text { Clinical and } \\
\text { Allied Health }\end{array}$ & Supervisor & \\
\hline 1 & 26 & 17 & 43 & 3 & 3 & 1 & 7 \\
\hline 2 & 39 & 33 & 72 & 2 & $1^{b}$ & 2 & 5 \\
\hline 3 & 34 & 37 & 71 & 2 & & & 2 \\
\hline 4 & 40 & 31 & 71 & 1 & 1 & 1 & 3 \\
\hline 5 & 27 & 23 & 50 & & $1^{c}$ & 1 & \\
\hline 6 & 43 & 27 & 70 & 2 & & 1 & 3 \\
\hline Total & 209 & 168 & 377 & 10 & 6 & 5 & 21 \\
\hline \multicolumn{8}{|c|}{ a: Five clients, three medical officers and an allied health professional that were approached did not participate } \\
\hline \multicolumn{8}{|c|}{ b: This interviewee worked in two communities } \\
\hline \multicolumn{8}{|c|}{ c: This interviewee had a dual clinical and supervisory role } \\
\hline
\end{tabular}

[The health worker] was always a little bit caught between the research project and then the demands of the clinic as well. (Medical officer: Community 1)

... we always have great plans [to support the model of care] and then basically we're a bit overrun by the clinic presentations. (Clinical nurse coordinator: Community 1 ) They're from the community so the community look to them for support and they can't exactly say "sorry, I am only here to look after this area and I can't attend to you". (Manager: Community 3)

The GBACC project sought to recruit experienced IHWs with Certificate IV Aboriginal Torres Strait Islander Primary Health Care so that they have the training and professional experience to be effective in aspects of their care coordination role that require independent activity. At two intervention sites, trainee IHWs were recruited when there were no alternative candidates. These workers participated in intensive chronic disease care clinical training to provide them with the skills to deliver health checks and care coordination. Industrial regulations in Queensland state that trainee IHWs require a higher level of supervision for client care planning and home visiting. ${ }^{17}$ If appropriate supervision was not available for trainees, these key elements of the model of care could not be implemented. This was reflected in the document review and in an interview with a supervisory health service staff member:

Unable to do home visit, need to be supervised. (GBACC IHW: Community 5)

So she was actually a trainee health worker here when she was put in this project so in some ways I guess she was set up to fail. (Nurse manager: Community 5)

The situation was resolved at one site when the trainee GBACC IHW resigned and a qualified worker was recruited to the position, and at the other site the IHW was successful in achieving IHW qualifications.

The Cairns-based ICST provided direction and support to the GBACC IHWs; however, dayto-day supervision for operational matters was the responsibility of local health service providers. While the document review did not reveal any confusion on the part of GBACC IHWs around their lines of management, health service supervisory staff expressed a view that there were problems associated with managing the health worker roles.

... and then there's the sort of line of management, so that she had two lines of 
management. One was through Cairns and the research project asking for things to be done, reports, figures ... (Medical officer: Community 1)

Iam (the IHWs) supervisor for everything else but the project. (Manager: Community 4)

This was problematic if the GBACC IHW was viewed differently by the local health service team despite efforts to integrate their role in the team.

... [the GBACCIHW] ispartof the teambutapart from the team. (Manager: Community 4)

... so other health workers or colleagues would sometimes see that if she's out doing home visits that might not be seen as being doing (sic) work or being helpful to the clinic. (Medical officer: Community 1)

\section{Training}

The Cairns-based customised group training delivered by the ICST to GBACC IHWs was designed to provide an understanding of the project objectives and the competencies to enable planning, delivery and coordination of chronic disease care for client caseloads. All GBACC IHWs completed each module in the training program.

Statements recorded in the IHW weekly reports indicate that the training was a key enabler for improving client engagement and access to services and for facilitating positive change in client behaviour. GBACC IHWs reported increased confidence in their knowledge and skills to deliver education, support and direction to their clients.

I have gained more confidence in my work and role now that I have found my footing and worked out how the whole system operates and it makes a huge difference when I find I can set out and go about my duties confidently. (GBACC IHW: Community 4)

They talked about innovative strategies they adopted to provide support, address barriers to care and achieve care goals. Examples of innovation by GBACC IHWs included developing goal-setting contracts for clients, constructing exercise equipment from drink bottles and sand, coordination of local transport to support home visits and using storytelling to explain health conditions and medicines.

Benefits of the training in building confidence of the GBACC IHWs were confirmed during stakeholder interviews.

The health workers were excited about learning... what it did was gave them confidence...to say "well, yes I know this and I can do this"... it gave them a new lease on life and a bit of excitement for their job.

(Allied health: Communities 2 and 3 )

However, local health service staff also talked about limitations to reinforcing learning from the Cairns training, due to what they perceived as poor communication about training delivered.

Idon't knowexactly what trainingshehad...I felt / suppose, you get trained, then you need follow-up of it. So it is possible to get trained and have that follow-up training here at the clinic because there are opportunities. (Medical officer: Community 1 )

I don't know what training was given... maybeifl had known ... it would have been helpful because I could have said "well, I'll supportyou. I'll help you with this". (Clinical nurse coordinator: Community 1)

That has been a bit of a limiting factor. I haven't been given feedback [about the training]. (Clinical nurse coordinator: Community 4)

The document review revealed that GBACC IHWs became frustrated when they were not able to "get on with the job" and complete clinical follow-up tasks and other duties. Many of the GBACC IHWs faced challenges using basic word processing, which was a barrier to completing planning, reporting, and resource development responsibilities. Lack of venipuncture skills were also raised as a barrier to progressing care for clients.

I would like to have (venipuncture training) happen. We might get a doctor or RN who is too overloaded for weeks at a time and I can have clients' pathology request forms sitting there for weeks. (GBACC IHW: Community 4)

Practical stuff was hindering her performance. Practical stuff was around pathology, simply because she couldn't do venipuncture. Then that was waiting time for patients. They get really annoyed with waiting. She had to wait for other staff to take bloods and rely on them. (Manager: Community 1 )

\section{Client engagement}

Client engagement includes issues that affect how clients connect with the GBACC IHW and the service. In their weekly reports, GBACC IHWs documented an increase in client engagement with the services as a result of regular follow-up.

One of the most positive points for me is that a client, who would not keep an appointment with the GP, health workers and $R N$ at the clinic, is now beginning to access all of the these health professionals. (GBACC IHW: Community 2)
A health service manager pointed out benefits to the clinic team when the GBACC IHW was able to engage clients in accessing chronic disease care.

... it makes my job easier because those particular clients that are on the GBACC, they're all up to date with their health issues, with their appointments... we don't have to worry, the clinic doesn't have to worry about that particular client or that particular referral ... (Manager: Community 6) Interviews with clients enrolled in the project generally spoke positively about it and their experience of the GBACC IHW.

... [the GBACC IHW] tells me how to handle [diabetes], how to use [medication]. It's really helpfulyou know. They tell me, explain to me and all that. Even Ilearn myself. If I see a book about diabetes or type 2 diabetes, I always read. (Client: Community 2)

[The GBACC IHW] told me to go there and she tell me everything, like what's happened, all those results, like them things, that I was happy myself. As long as I know, like how's my body. (Client: Community 3)

Yeah it's got better [since GBACC]. Ijust start walking everywhere. Well you know when [the GBACC IHW] wasn't around I couldn't walk much. I sad ah ... and I went then and see [the GBACC IHW]. I say "What's good food?" He tells me all. He tell me don't have white bread and all that. And I asked for butter. "What sort of butter?" He showed me the picture and I bought that same butter from the shop. I feel real good. (Client: Community 6)

The document review revealed that GCACC IHWs experienced problems with client engagement similar to other members of the PHC team, but they were able to articulate reasons for non-engagement. A range of factors that were barriers to client engagement were documented in weekly reports. They included: clients are working, sorry business, family issues, client dissatisfaction with the service, community events, difficulty in finding people for follow-up and lack of interest/response from participants.

Some clients are at work. (GBACC IHW: Community 3)

... was tired of having different doctors.

(GBACC IHW: Community 4)

Community activity happening, clients get slack to come down Health Centre. (GBACC IHW: Community 1)

A health service provider expressed a view that some community members did not access services because they are resigned to 
the fact they will develop diabetes and there is little that can be done about it.

Some young people think that no matter what Ido, I will eventually get it because mum had it, and grandma had it, sol must be going to get it. (Generalist IHW: Community 1)

\section{Clarification of IHW care coordination role}

The document review did not reveal any issues with the GBACC IHWs' understanding of their role. They appeared to be clear about what was expected of them.

I see a lot deeper into each of our clients and it's wonderful how they discuss anything with me and we work as a team in partnership to achieve what we plan together. (IHW: Community 4)

Health service managers and supervisors also demonstrated clear and positive views about the role of the GBACC IHWs.

A dynamic person, someone who can think on their feet, someone who can run on their feet. You know, it's not a laid-back role, it's a 'get out there, get into it' role. (Manager: Community 6)

Lookyou can't do it without a health worker. You know the cultural mentoring side of that role is huge, let alone the clinical. [The GBACC IHW role is] more education and more cultural mentorship. (Manager: Community 6)

[The GBACC IHW] was able to monitor them more stringently and she could monitor them in theirhome... She could sit down and discuss a lot more individual needs. I think we'd be lost without theircultural knowledge... it was concentrated care on the people concerned and they seemed to be happy to be on the project. They can actually take people aside and explain to them what it's about and get them to understand in their own language and things like that, what it means for them. (Nurse manager: Community 5)

When other team members had a good understanding of the GBACC IHW role, this became an enabler for them to be effective in care coordination.

\section{Infrastructure}

The provision of high quality chronic disease care relies on health service infrastructure including client information systems, adequate clinical space and equipment, as well as community infrastructure such as access to transport.

Lack of access to transport to support home visits or health services within or outside the community was a common problem for all GBACC IHWs.
Clients who had appointments or surgery booked... has been cancelled due to ferry's mechanical problems. (GBACC IHW: Community 3)

No public transport (GBACC IHW community 1), no vehicle (GBACC IHW community 5) and no transport. (GBACC IHW community 4)

However, lack of transport infrastructure was not considered a problem by everyone.

Should be walking anyway. I tell them they should be walking - if they can walk up to gamble, they can walk to the health centre. (Generalist IHW: Community 1)

All the GBACC IHWs reported issues with electronic patient information systems. Most were related to information technology problems and slow systems; however, in three communities, GBACC IHWs had no access to the patient information system used by medical and nursing staff because there were a limited number of software licences per site. Clinical team members at one site were able to assist with access to the clinical information systems when required, but only limited opportunistic access was possible at the other two sites.

At a fourth site, difficulties arose due to organisational agreements between various health service providers.

[The GBACC IHW] has access to the [paper medical] files, yes, and that's in the [organisational] agreement, but not to [the electronic information system] and it's been contentious. (Clinical nurse coordinator: Community 4)

Being unable to electronically record daily activities and episodes of client care led to other PHC clinicians concluding that some routine client follow-up remained outstanding.

... we've had duplication of [client] invitations, clients getting confused and I guess that's what's led to where we are now. (Clinical nurse coordinator: Community 4)

Lack of access to private clinic space to meet clients was a barrier to care for the GBACC IHWs and their colleagues.

The doctor left early after lunch due to being frustrated by the fact he had no available room in which to see to clients. (GBACC IHW: Community 4)

Access, though, I mean where will she sit?... clinical space here-this clinic was designed for 60 people and we have one treatment room, one consulting room - and now we have 300 regular clients. (Clinical Nurse coordinator: Community 4)

\section{Team approach to care}

The GBACC model of care aimed to promote GBACC IHWs as key members of the multidisciplinary chronic care team with a defined role to support care coordination of complex clients. This was achieved with mixed levels of success.

In two communities, medical officers were unwilling to collaborate to complete care plans and team care arrangements. This was a major barrier to GBACC IHWs facilitating care coordination. In lieu of care plans, the GBACC IHWs used the standard clinical guidelines to plan care needs for clients. However, requests to clinicians to complete care requirements were not always responded to positively. This was perceived as lack of professional respect by GBACC IHWs in their weekly reports. On occasion, clinicians rejected their requests to order routine investigations, despite the recommendations being consistent with Queensland Health Chronic Disease Guidelines.

The doctor said the client didn't need an ECG as he had no symptoms. (GBACC IHW: Community 2)

It was stressful for the GBACC IHWs when they saw that their clients or other community members were not receiving recommended care.

If it weren't for this project some clients would fall through the cracks. (GBACC IHW: Community 2)

In most communities, positive engagement with other PHC team members was evident.

You don't know how good it is to know the person more, to sit with them and understand. (Medical officer's feedback to GBACC IHW about a home visit they attended together: Community 4)

Ithought that the health worker'sjudgment on how someone was travelling and their priority issues was very good and I found the health worker to be a real resource and a real help to my work. (Medical officer: Community 1 )

Oh completely [trusted the clinical judgement of the GBACC IHWs]. I had already established a relationship with them and I knew I could trust them, but this took them into another level of assessment and judgement. So yeah, they were good. (Allied health: Communities 2 and 3 )

\section{Discussion}

The key objective of the GBACC project was to implement a model of intensive 
case management for clients with chronic disease. GBACC IHWs were trained to deliver education, support, clinical care and care coordination to a defined group of clients. The process evaluation explored the experience of implementing the GBACC model of care from different perspectives and identified a number of issues that explain why the intervention was not fully implemented, particularly in the important area of care coordination. Barriers included demands on the IHWs outside their chronic disease care coordination role and chronic care plans not being developed for all participants to direct care needs. This suggests that the case management model of care was not fully integrated within existing services as intended.

Key enablers to effective care coordination by IHWs integrated in the GBACC model were: provision of training to support the IHW role; communication of the IHWs role to the individual workers and their colleagues; IHWs knowledge of their clients and environment; and ongoing support by the ICST. Collectively, these strategies increased IHWs' confidence and capacity to provide chronic disease care and service coordination. The training appeared to address the significant barrier to IHWs developing their role in chronic disease care identified in the Northern Territory. ${ }^{4}$ Key barriers to care coordination identified by the GBACC IHWs included: clinic staff shortages and lack of a consistent team with accessible medical and allied health staff; working with staff whose practices did not always adhere to chronic care guidelines; lack of awareness by clinical colleagues around training and competencies of the GBACC IHW; and infrastructure issues, in particular, those that impede access to a common patient information system. Except for infrastructure and clinic staff shortages, which were issues common to all sites, the reported barriers primarily related to state government service sites. The community-controlled service model is based on a philosophy that the IHW is a key member of the multidisciplinary team and central to facilitating access to locally based and visiting clinicians.

In a systematic review to assess the effectiveness of community health workers in supporting the care of diabetic clients, Norris et al. ${ }^{12}$ found it was difficult to determine which intervention components led to positive outcomes. They did establish that improved client physiological outcomes were related to the health worker being involved in clinical care, liaising to give assistance to other health staff to provide care and having a direct client teaching role. The role of IHWS in improving access to services was confirmed in a recent study from the Northern Territory where Aboriginal and Torres Strait Islander patients reported that they preferred to receive services from Indigenous workers who understood their language and community. ${ }^{18}$ The GBACC model incorporated these elements into the role of the IHWs as part of their case management. The process evaluation confirmed implementation of these elements of the model of care, but it was not always easy to maintain. Significant organisational change and restructuring within the Queensland Department of Health, high staff turnover and limited orientation of new staff were all factors that affected the GBACC IHWs' ability to be effective. High staff turnover and limited orientation are often factors that are identified as barriers to implementing the chronic care model in remote Indigenous communities in Australia. ${ }^{19,20}$ Despite the organisational difficulties, a high level of satisfaction was reported with their role by the GBACC IHWs and by many clients about delivery of care.

Norris et al. suggest that to understand how much community health workers contribute to improved health outcomes for clients with diabetes, researchers need to identify appropriate settings for the care, as well as the health workers' optimal role in care provision. ${ }^{12}$ The systems assessment tool, derived from the MacColl $\mathrm{CCM}^{7,21}$ and modified for application in the Australian Indigenous primary health context by the Audit and Best-practice for Chronic Disease (ABCD) project, ${ }^{22,23}$ provides a useful framework to describe the care delivery system required to support chronic disease management in the Indigenous health context. The tool prescribes the need for integration of Indigenous knowledge into clinical practice and community based activities as well as cultural competency among staff. It recommends appropriateness of a health setting or system be assessed against the following broad domains: 1) delivery system design; 2 ) information systems and decision support; 3) selfmanagement support; 4) links with the community, other health services and other services and resources; and 5) organisational influence and integration.

In their daily practice, the GBACC IHWs seem most able to strengthen systems for chronic disease care in domains where they are able to influence practice, based on their skills and knowledge of the community and their local health system. These domains are client self-management support, and linking with community and other health services, and other services and resources. A systematic review of the effectiveness of the chronic care model to improve diabetes care highlighted that improving the delivery system design is one of the most important components in achieving improved diabetes care. ${ }^{8}$ In the GBACC study, there appeared to be a number of barriers inherent in the organisation of the health systems and delivery system design that prevented individual clients, health staff, families and communities from reaching their full potential in accessing and providing effective chronic care. This would suggest that more work is required to improve clarification of the IHW's role with team members and consistent engagement with medical officers to complete care planning.

Analysis of chronic disease care through the lens of continuous quality improvement by the $A B C D$ project found system weaknesses similar to barriers to care coordination in the GBACC project. They included: understaffing generally as well as underfunding for IHW positions; poor training of IHWs; irregularity of doctors and frequent new nurses; lack of communication among teams; poor coordination of the work of doctors, nurses and IHWs; complexity of clinical information systems and lack of information technology maintenance; and having to prioritise acute care demands. ${ }^{424,25}$ The ABCD evaluators suggested that chronic care quality and client outcomes improvement could be achieved by shifting the focus of systems thinking away from individual clinicians and towards the broader health systems they work in.

A key challenge for IHWs was integrating case management into the usual approach to care in an environment where acute care demands take priority. GBACC IHWs were part of the PHC team and employed by the local service to promote integration into existing service delivery systems, but with a quarantined role to ensure that care coordination was delivered. There was an assumption in the project design that the $\mathrm{PHC}$ teams were supportive of this change because the concept was welcomed at the organisational level and the IHWs were an additional resource to $\mathrm{PHC}$ teams. This may not have been the case. Change management theory and key learnings from the implementation of CQI to improve chronic disease care would suggest that a greater emphasis on engaging 
with clinical leaders and local champions would have assisted with improved team work, ${ }^{23}$ but this is challenging to maintain in an environment of high staff turnover.

Maintaining commitment to chronic disease strategies requires strong leadership and ongoing organisational commitment. ${ }^{25}$ This was difficult to achieve in many sites due to the organisational change to the Queensland Department of Health that directly affected five of the six communities in the study. Frequent turnover of management and clinical staff, a significant reduction in generalist IHWs working in three sites and frequent absences of IHWs due to training or family reasons were all reasons why it was difficult to maintain the commitment of dedicating a worker to chronic disease care coordination. These factors need to be addressed to achieve best practice care for remote Aboriginal communities. ${ }^{19}$

The GBACC process evaluation was an opportunity to strengthen our understanding of the RCT results, and to improve the model of care prior to broadening the intervention across six wait-list communities. Limitations of the face-to-face interviews include the small sample size, particularly in relation to the clinical service providers; however, this reflects the size of the workforce in the study sites. Two clinician participants worked across two study sites and were able to provide perceptions on both. Additionally, it was unfortunate that, apart from one study site, medical officers were unavailable or declined to participate. At the same time, there was a great deal of consistency between information provided by clinicians and supervisors, irrespective of professional role, and it was felt that data saturation was reached. Selection bias in client interviews was evident because clients who anecdotally proved difficult to followup clinically were equally difficult to access as interview participants. This important group would potentially add a great deal to our understanding of failures in client engagement and service delivery.

In summary, implementation of intensive case management by IHWs in this trial was suboptimal, which could partly explain the modest clinical impact. There was good engagement with medical officers in four of the six sites and all sites implemented care coordination by IHWs. However, in more than half of the implementation sites there were significant barriers to the effective performance of the IHW in their role. These included competing demands of acute care, lack of understanding by the team of the GBACC IHW role, lack of respect for the cultural role and training of the GBACC IHWs, poor utilisation of work space, ineffective team work (including sharing of the electronic patient records) and failure to follow chronic disease guidelines.

\section{Conclusion}

Placing a skilled and dedicated IHW to improve chronic care and care coordination is insufficient to improve chronic disease outcomes in the absence of a supportive service model. The barriers that the GBACC IHWs faced are mostly systems issues and were not a reflection of their competence or capacity to engage with clients or with their communities.

Many PHC services continue to operate in response to acute episodic conditions and, as such, are not always oriented to cope with the growing need for chronic care. The morbidity and mortality from chronic illness is increasing in all Australian communities, and health care strategies to slow disease progression and prevent complications are important. Provision of acute care is essential; however, PHC services should be adequately resourced and designed to provide regular and extended treatment for people suffering from complex chronic conditions. In Indigenous communities, the PHC model may be re-oriented to include the concept of a dedicated role for care coordination as part of an integrated service delivery model and actively support the unique contributions of IHWs to chronic care and care coordination.

\section{References}

1. Vos T, Barker B, Begg S, Stanley L, Lopez AD. Burden of disease and injury in Aboriginal and Torres Strait Islander Peoples: The Indigenous health gap. Int $J$ Epidemiol. 2009;38(2):470-7.

2. Gracey $M$, King $M$. Indigenous health part 1 : Determinants and disease patterns. Lancet. 2009:374(9683):65-75.

3. McDermott RA, Schmidt BA, Sinha A, Mills P. Improving diabetes care in the primary healthcare setting A randomised cluster trial in remote indigenous communities. Med J Aust. 2001;174(10):497-502.

4. Si D, Bailie R, Cunningham J, Robinson G, Dowden $M$, Stewart $A$, et al. Describing and analysing primary health care system support for chronic illness care in Indigenous communities in Australia's Northern Territory- use of the chronic care model. BMC Health Serv Res. 2008;8(1):112.

5. Queensland Health. Chronic Disease Guidelines. Brisbane (AUST): State Government of Australia; 2010. p. 43.

6. Central Australia Rural Practitioners Association. CARPA Standard Treatment Manual. Alice Springs (AUST): CARPA; 2009.
7. Improving Chronic Illness Care. The Chronic CareModel [Internet]. Seattle (WA): MacColl Centre for Health Care Innovation; 2006 [cited 2014 May 15]. Available from: http://www.improvingchroniccare.org/index. php?p=Model_Elements\&s $=18$

8. Si D, Bailie R, Weeramanthri T. Effectiveness of chronic care model-oriented interventions to improve quality of diabetes care: A systematic review. Prim Health Care Res Dev. 2008;(9):25-40.

9. World Health Organisation. Innovative Care for Chronic Conditions: Building Blocks for Action. Geneva (CHE): WHO; 2002.

10. Wagner EH, Austin BT, Davis $C$, Hindmarsh M, et al. Improving chronic illness care: Translating evidence into action. Health Aff. 2001;20(6):64-78.

11. Abbott $P$, Gordon $E$, Davison J. Expanding roles of Aboriginal health workers in the primary care setting: Seeking recognition. Contemp Nurse. 2008;26(1):66-73.

12. Norris SL, Chowdhury FM, Van Le K, Horsley T, Brownstein JN, Zhang $X$, et al. Effectiveness of community health workers in the care of persons with diabetes. Diabet Med. 2006;23(5):544-56.

13. Perry HB, Zulliger R, Rogers MM. Community health workers in low-, middle-, and high-income countries: An overview of their history, recent evolution, and current effectiveness. Annu Rev Public Health. 2014:35:399-421.

14. Schmidt B, Wenitong M, Esterman A, Hoy W, Segal L, Taylor S, et al. Getting better at chronic care in remote communities: Study protocol for a pragmatic cluster randomised controlled of community based management. BMC Public Health. 2012;12(1):1017.

15. McDermott R, Schmidt B, Preece C, Owens V, Taylor S, Li $\mathrm{M}$, et al. Community health workers improve diabetes care in remote Australian Indigenous communities: Results of a pragmatic cluster randomized controlled trial. BMC Health Serv Res. 2015;15:68.

16. Wilkinson D, Mott K, Morey S, Beilby J, Price K, Best $\mathrm{J}$, et al. Evaluation of the Enhanced Primary Care (EPC) Medicare Benefits Schedule (MBS) Items and the General Practice Education, Support and Community Linkages Program (GPESCL) Final Report. Canberra (AUST): Commonwealth Department of Health and Ageing, 2003.

17. Queensland Government. Aboriginal and Torres Strait IslanderHealth WorkerCareerStructure. Brisbane (AUST): State Government of Queensland; 2009.

18. Aspin C, Brown N, Jowsey T, Yen L, Leeder S. Strategic approaches to enhanced health service delivery for Aboriginal and Torres Strait Islander people with chronic illness: A qualitative study. BMCHealth Serv Res. 2012;12:143.

19. Gador-White A, Wakerman J, Campbell D, Lenthal S, Struber J, Hope A, et al. Cost of best practice primary care management of chronic disease in a remote Aboriginal community. Med JAust.2014:200(11):663-6.

20. Si D, Bailie R, Togni S, D"Abbs P, Robinson G. Aboriginal health workers and diabetes care in remote community health centres: A mixed method analysis. Med J Aust. 2006;185 (1):40-5.

21. Bonomi A, Wagner E, Glasgow R, Von Korff $M$ Assessment of chronic ilness care: A practical tool for quality improvement. Health Serv Res. 2002;37(3): 791-820.

22. Si D, Bailie R, Cunningham J, Robinson G, Dowden $M$, Stewart $A$, et al. Describing and analysing primary health care system support for chronic illness care in Indigenous communities in Australia's Northern Territory- use of the Chronic Care Model. BMC Health Serv Res. 2008;8(1):112.

23. Gardner KL, Dowden M, Togni S, Bailie R. Understanding uptake of continuous quality improvement in Indigenous primary health care: Lessons from a multisite case study of the Audit and Best Practice for Chronic Disease project. Implement Sci. 2010;5:21.

24. Bailie RS, Robinson G, Kondalsamy-Chennakesavan SN, Halpin S, Wang Z. Investigating the sustainability of outcomes in a chronic disease treatment programme. Soc SciMed. 2006;63(6):1661-70.

25. Bailie R, Si D, Dowden M, O'Donoghue L, Connors C, Robinson $\mathrm{G}$, et al. Improving organisational systems for diabetes care in Australian Indigenous communities. BMC Health Serv Res. 2007;7:67. 